\title{
Validation of a photoplethysmographic heart rate monitor: Polar OH1
}

\author{
E. Hermand ${ }^{1}$, J. Cassirame ${ }^{2,3}$, G. Ennequin ${ }^{4}$, O. Hue ${ }^{1}$
}

${ }^{1}$ Laboratoire ACTES, EA3596, Université des Antilles, Pointe-à-Pitre, Guadeloupe, France

${ }^{2}$ EA 4660 "Sport Culture et société" \& Exercise, Performance, Health, Innovation Platform, Bourgogne Franche-Comté University, France

${ }^{3}$ EA 7507, Laboratoire Performance, Santé, Métrologie, Société. Reims, France

${ }^{4}$ Pepite EA4267, EPSI, Bourgogne Franche-Comté University, France

\section{Original investigation.}

\section{Corresponding author:}

Eric Hermand

Laboratoire ACTES ('Adaptations au Climat tropical, Exercice et Santé')

Université des Antilles, Pointe-à-Pitre, Guadeloupe

Email : eric.hermand17@gmail.com

Mailing address: Laboratoire ACTES, UFR STAPS, Université des Antilles, Campus de

Fouillole, BP 250, 97157 Pointe-à-Pitre Cedex

The Laboratories ACTES (EA3596) and EPSI (EA4660) conducted this study independently and unbiased for Polar Electro Oy.

The authors declare no conflicts of interest.

Abstract word count: 249

Text-only word count : 2488

\section{Keywords :}

Photoplethysmography

Accuracy

Exercise

\section{Bland-Altman}

Training load 


\section{Abstract}

This study assessed the validity of a photoplethysmographic heart rate (HR) monitor, the Polar OH1 in various sports performed in ecological conditions: running, cycling, soccer, kayaking, walking, tennis and fitness. Seventy trained athletes (56 males, 14 females) wore the Polar $\mathrm{OH} 1$ armband and the H7 chest belt during training. A total of 390 hours and 38 minutes of recording were compared using a 20-bpm window to assess dataquality, and. Bland-Altman agreements and ICC analyses to test accuracy. Linear regression analyses evaluated the HR accuracy and correlation with skin tone. Training loads (TRIMPs) were compared for each session. Reliability was high for endurance sports (>99\%) and lower for sports involving arm movements (92 95\%). Biases were slightly negative for all sports, whereas widths of limits of agreement varied from 7 to $20 \mathrm{bpm}$. Bland\&Altman agreements were all under $5 \%$ except tennis, kayak and fitness. HR accuracy was positively correlated to skin tone $(\mathrm{p}<0.05)$. Finally, TRIMPs from $\mathrm{OH} 1$ device were inferior to criterion's (except walking and soccer), within a $3 \%$ range from reference. Hence, $\mathrm{OH} 1$ represents a valid tool to monitor instantaneous HR and training load, especially for endurance sports. 


\section{INTRODUCTION}

Over the past decades, heart Rate Monitor (HRMs) have become popular tools for training guidance and physical activity monitoring [1,2] . These devices are regularly upgraded with improving technology (GPS integration, inertial sensor, memory) to respond to user requirements [3]. The first commercial HRMs based heart rate (HR) measurement on thoracic electrical measurement using chest belts to obtain ECG signal transduced in bursts when R peaks occur. More recently, manufacturers have equipped their devices with a photoplethysmography (PPG) system behind the wrist clock, as chest belts are less convenient for women and less hygienic.

Photoplethysmography was first used in the late 1930s [4] especially in the medical field for measuring $\mathrm{HR}, \mathrm{O}_{2}$ saturation and cardiac output [5]. In brief, a diode emits a single wavelength light which is propagated through the tissue of interest and then is captured by a distant detector. The analysis of the temporal difference between the signal source and collection provides the required information.

PPG is mainly used with two light wavelengths. Red light is common in medical and hospital technologies, whereas green LEDs have gained popularity for consumer products. Compared to red light, they show higher sensitive to skin melanin and shallower data acquisition but much greater affinity to oxyhemoglobin and deoxyhemoglobin [6], which means better accuracy in HR monitoring [7]. Green light monitors are therefore less subject to movement artifacts than red light monitors [8], and the low cost of green light technology has gradually made them available to the general public, especially for HR-monitored activities, like exercise and sports activities. Major brands have developed wrist-worn watches designed for both recreational and trained athletes, integrating an optical HR (OHR) monitor in the watch case, to replace the traditional chest belt. The HR values of OHR are insufficiently accurate [9-13], despite recent improvements in the number of usable light emitters and the built-in algorithms [14,15]. Among the many reasons are mainly low skin temperature [16], motion artifacts [16,17], inadequate attachment or positioning $[13,16,18]$, skin type $[14,15]$, activity type [19] and intensity of exercise $[9,19,20]$. Another reason is the number of LED sources: most of brands use two or three light sources, which may be insufficient to retrieve blood flow data, especially through the bony wrist region, more sensitive to motion artifacts [17]. Most of the aforementioned studies validating OHR monitors were performed in laboratory or controlled environments like sports gyms [9,14,15,21,22], using standardized protocols of low-to-medium intensity and limited duration (typically $<1$ hour) $[9,15,21-23]$. From a more training-centered perspective, these systematic protocols might not be valid for use in 
ecological conditions, i.e., outdoor or with varying intensities and durations, for team sports (e.g., soccer) or endurance sports (e.g., running and cycling) at regional or national levels requiring daily training load monitoring [16].

The accuracy and reactivity of these devices are essential to HR measurement. Currently, HR measures are mainly used to calibrate training intensity with real-time HR values and calculate training load $[1,2]$. In the first case, athletes use the HR on the wrist display to adjust speed or other mechanical variables. Hence, accuracy and reactivity are both important for proper intensity calibration. In the second case, collected HR values are computed with various methods to obtain a global training load, such as TRIMP by Banister et al. [24,25], which remains the gold standard. Inaccurate HR measurement yields incorrect TRIMP calculation $[24,26]$, which means that race day fitness level and fatigue are also likely to be incorrectly assessed $[24,27]$.

Recently, Polar Electro Oy (Kempele, Finland) developed a 6-LED wrist clock (M600) producing a valid HR signal in most (controlled) conditions [14]. Nevertheless, Horton et al. point out that activities like weight lifting, muscle and ligament tension in the wrist may interfere with HR detection from capillary blood flow [14]. More recently, the same manufacturer designed an autonomous 6-LED OHR sensor, the OH1, that can be strapped around the arm or forearm and is potentially less subject to the motion artifacts of wrist-worn watches. Its use was validated for moderate-intensity sports activities [21].

The aim of this study is (1) to assess the accuracy of the HR signal in trained athletes wearing Polar $\mathrm{OH} 1$ monitor in ecological conditions during outdoor activities, including team and endurance sports, compared to traditional chest belt (Polar H7, Polar, Electro Oy, Kempele, Finland), and (2) to compare training load obtained from the HR calculation provided by the two devices.

\section{METHODS}

Subjects. 70 subjects, 56 males and 14 females, participated in this study. All were in good physical condition and exercised regularly, from 5 to 20 hours per week, during personal free time or with a club. Mean $( \pm$ SD) age, height and body mass of all participants were $19.7 \pm 5.8$ yrs, $174.4 \pm 10.5 \mathrm{~cm}$, and $66.9 \pm 12.1 \mathrm{~kg}$, respectively. The skin types of athletes living in Burgundy (France, 23 participants) and the West Indies (47) were assessed with the Fitzpatrick skin scale [28], from 1 (lightest tone) to 6 (darkest). 


\section{Experimental protocol and data collection.}

123 Participants followed their usual training routine following coaches' instructions. Running, biking and walking were performed on various terrains, switching between flats, hills and downhills, which induced a wide HR spectrum from low to high. Tennis, crossfit and soccer were performed on flat ground or in water, but also showed low and high HRs due to successive pauses and sudden accelerations or repetitions.

128 For each session, participants wore two HR monitors: the Polar OH1 monitor and the Polar H7 belt (Polar Electro Oy, Kempele, Finland) as the criterion measurement [12,29], paired with a Polar M400 watch. Following the manufacturer's instructions, the OH1 device was strapped around the upper arm, firmly enough to remain in place but not enough to obstruct blood flow. Recordings for both were started at rest before the exercise start and terminated after a short recovery time. Procedures were conformed ethics in sport and exercise science [30].

\section{Data processing and statistical analyses.}

HR data from both M400-H7 and OH1 were retrieved from the Polar Flow web service, visually inspected for criterion dysfunction, discarded when necessary, and exported as spreadsheets. For comparisons of each measurement session, signals were synchronized with the least square method and smoothed on a 10-s window.

First, OHR quality was defined as the percentage of data within $\pm 20 \mathrm{bpm}$ of the Polar H7 signal (OH1-IN), with data outside this range labeled OH1-OUT. Second, OHR accuracy was assessed with Bland-Altman analysis [31] to test agreement between $\mathrm{OH} 1$ and $\mathrm{H} 7$ data: bias (mean difference, MD), standard deviation (SD) and upper and lower limits of agreement (LOA, defined as $\mathrm{MD} \pm \mathrm{SD}$ ) were calculated. Last, minimal, maximal and average HRs were calculated and training load values were computed following Banister's modified TRIMP [26], using theoretical or measured rest and maximal HRs. Intraclass correlation coefficients (ICCs) [32] were computed using $\mathrm{OH} 1$ and $\mathrm{H} 7$ data for each sport: their value indicates the reliability of the $\mathrm{OH} 1$ measures vs. criterion (<0.5: poor, 0.5-0.75: moderate, 0.75-0.9: good, >0.9: excellent) [33].

For the skin tone effect on HR accuracy, linear regressions established potential correlations between these discrete quantitative values and biases. All parameters including the training loads obtained from the $\mathrm{OH} 1$ and $\mathrm{H} 7$ sensors from each session were compared using Student tests. A p-value $<0.05$ was considered significant. 
157 Examples of the simultaneous HR recordings from the $\mathrm{H} 7$ and $\mathrm{OH} 1$ are presented in Figure 1; the right figure illustrates the dropout phenomenon outside the 20-bpm criterion zone. In all, 390 hours and 38 minutes of recordings were analyzed (cycling: 113hr,49min; running:102hr,26min; soccer: 47hr,45min; kayaking: 50hr,41min; walking: 37hr,30min; tennis: $18 \mathrm{hr}, 35 \mathrm{~min}$; and fitness: $19 \mathrm{hr}, 53 \mathrm{~min}$ ), distributed across 233 sessions. Differences in sports durations were mainly due to the much longer duration of walking and cycling sessions (up to several hours). Results are compiled in Table 1.

Sports involving more vigorous upper limbs movements (kayak, tennis and fitness) exhibited wider LOAs than endurance sports (cycling and running), whereas biases were not impacted by activity type (Fig. 2 and Table 1). ICCs for all sports were above 0.99 (Table 1), indicating the excellent reliability of $\mathrm{OH} 1$ data vs. criterion.

The mean value of skin type was $3.4 \pm 2.2$ and it affected bias and LOAs. The correlation between skin tone and bias was positive $(\mathrm{p}<0.001)$, i.e., accuracy (bias) was decreased with darker skin.

Training loads were not different in walking and soccer, but they were systemically higher with the criterion $\mathrm{H} 7$ monitor for other sports (Table 2).

\section{DISCUSSION}

Although most studies have sought to validate similar HR devices in a laboratory environment, our research was based on data collected in real-life situations by athletes following their training routines in various type of activities.

Our first result was the difference in the proportion of data outside the threshold limits - i.e., $20 \mathrm{bpm}$ from criterion value (OH1-OUT) - between the arm-driven sports (tennis, kayaking and fitness) and non-arm-driven sports. The latter showed a very high percentage of useful values in endurance sports like walking, running and cycling, whereas the upper-body based sports presented a much higher error rate (Fig. 1B and 1C, Table 1). In the absence of dedicated tools to assess this imprecision, we can only assume that this was due to motion artifacts from the arm and chest movements, as reported by others $[12,14,16]$. As a corollary, these three sports also provided the widest LOAs, yet still less than those of the same brand wrist-worn model [14]. Nevertheless, the $\mathrm{OH} 1$ provided less than $1 \%$ data out of threshold in the traditional endurance sports (cycling, running, walking) and soccer (Table 1), which represents a very decent number for athletes and coaches relying on HR data. Although the 
algorithm to extrapolate HR remains Polar-proprietary, we can nevertheless assume that the 6-LED system provides reliability superior to that of the traditional 2- or 3-LED devices [14,15]: subcutaneous blood information transmitted and collected through six light sources and captors is centrally analyzed, and therefore erroneous data is better detected and discarded. From the synchronized HR signals, we also note that the OH1 HR values were regularly slow to increase or decrease during intensity variations (Fig. 1C). Technically, this phenomenon can be related to delays in microvascular blood flow increases or the smoothing filters integrated into the $\mathrm{OH} 1$ device to avoid large errors in HR measurement. Hence, in intermittent exercise, this phenomenon can enlarge LOAs because of the shift in instantaneous HR values collected by the two systems.

In addition, as noted above, the sports showed discrepancies. Sports involving active use of arms (tennis, fitness, kayaking) led to decoupling mainly in the transitions from low to high HR, for example during acceleration (Fig. 1B), and several minutes might have been necessary for the system to readjust properly. A similar phenomenon was recently observed in more controlled conditions with another wristwatch from the same brand [14]. On a side note, a non-negligible number of recordings could not be analyzed due to criterion dysfunction, mostly in kayaking, as vigorous chest movements impaired HR detection by the $\mathrm{H} 7$ belt. As the standardized positioning of the $\mathrm{OH} 1$ on the upper arm also presented motion artifacts, it might be better to strap it to body limb less subject to data collection failure.

Similarly, biases were systematically greater in arm-driven sports, though they remained under $1 \mathrm{bpm}$ and negative, implying that the $\mathrm{OH} 1$ measures a (very slightly) lower HR than the criterion (Table 1), as noted by others $[14,19,21,34]$ or not $[16,35]$. They also had larger Bland-Altman agreements, although just above 5\% for tennis and kayaking.

Mean HRs measured by the $\mathrm{OH} 1$ were lower for all sports except walking and soccer, but the difference was limited to a gap less than $1 \mathrm{bpm}$ (Table 1), as observed for other devices $[18,19]$. Yet, the minimal and maximal measured HR values showed no difference, and these undervalued data are still reliable markers in detecting overtraining risks [1]. Therefore, differences in mean HR could lead to errors in the computation of training load, as athletes and coaches collect and analyze these valuable data to assess fitness peaks and tapering periods [36]. Indeed, according to the HR data, the TRIMP values extrapolated from the $\mathrm{OH} 1$ device were lower than those of the criterion (Table 2) except for walking and soccer, but the difference remained small, from $3 \%$ for kayaking to $0.7 \%$ for cycling. Therefore, $\mathrm{OH} 1$ TRIMP may be used for training endurance athletes (cyclists and runners), who mostly use it 
in their training program[26]. To our knowledge, no studies on OHR have focused on training loads for confirmed athletes. Overall, the ICCs confirmed the excellent correlations between $\mathrm{OH} 1$ and criterion HR for all sports studied here (Table 1).

Interestingly, at times the $\mathrm{OH} 1$ provided apparently trustworthy when criterion failed. For example, several kayaking recordings were discarded because of Polar $\mathrm{H} 7$ dysfunction underwater (rolling) or during higher exercise intensities (maximal or submaximal intervals) when the $\mathrm{H} 7$ belt did not remain properly strapped around the chest [37]. The same observation were made in swimming, and $\mathrm{OH} 1$ accuracy should be evaluated in aquatic sports against a validated criterion [38]. Similarly, when soccer players chested the ball, it occasionally displaced the HR belt and disrupted HR data, whereas the OH1 provided accurate HR values (Table 1).

Our large and various sample from the West Indies and Burgundy enabled us to compare the effect of skin tone on HR accuracy. As have other studies, our study confirms that biases become larger with darker skins $[9,15]$, though this was negligible. Nevertheless, the biases were notably very low in soccer, where more than $90 \%$ of players (Table 1) from the West Indies exhibited values on Fitzpatrick skin scale equal to or above 5, which does not agree with previous conclusions $[9,15]$. A plausible explanation might be West Indies' hot and humid climate, which could have induced greater peripheral vasodilation [39], thereby making the blood signal more accessible to the $\mathrm{OH} 1$ sensor, regardless of skin tone.

This device is also useful for daily heart rate monitoring in patients to assess, for example, daily energy expenditure [40], particularly in overweight and pregnant women, whose tolerance and acceptability of chest strap constriction are lower [41]. An OHR device strapped around the arm or forearm would supposedly be better tolerated.

In conclusion, the Polar $\mathrm{OH} 1$ was worn by athletes and studied in field conditions. It showed good overall reliability for all activities, especially traditional endurance sports like running and cycling. It might therefore be a reliable alternative to constrictive chest strap for regular and intensive training. In that matter, multiple emitters ( 6 in the Polar $\mathrm{OH} 1$ ) certainly play a key role. However, sports implying chest and arm movements induce a higher rate of errors and heart rate dropouts. In the future, studies will be needed to assess its accuracy for water sports, and interesting potentialities should be explored in health tracking. 


\section{References}

1 Achten J, Jeukendrup AE. Heart rate monitoring: applications and limitations. Sports Med 2003; 33: 517-538

2 Schneider C, Hanakam F, Wiewelhove T, Döweling A, Kellmann M, Meyer T, Pfeiffer M, Ferrauti A. Heart Rate Monitoring in Team Sports-A Conceptual Framework for Contextualizing Heart Rate Measures for Training and Recovery Prescription. Front Physiol 2018; 9: 639

3 Cardinale M, Varley MC. Wearable Training-Monitoring Technology: Applications, Challenges, and Opportunities. Int J Sports Physiol Perform 2017; 12: S2-55-S2-62

4 Hertzman AB. The blood supply of various skin areas as estimated by the photoelectric plethysmograph. American Journal of Physiology-Legacy Content 1938; 124: 328-340

5 Allen J. Photoplethysmography and its application in clinical physiological measurement. Physiol Meas 2007; 28: R1

6 Van Kampen EJ, Zijlstra WG. Determination of hemoglobin and its derivatives. Adv Clin Chem 1965; 8: 141-187

7 Lee J, Matsumura K, Yamakoshi K, Rolfe P, Tanaka S, Yamakoshi T. Comparison between red, green and blue light reflection photoplethysmography for heart rate monitoring during motion. Conf Proc IEEE Eng Med Biol Soc 2013; 2013: 1724-1727

8 Maeda Y, Sekine M, Tamura T. Relationship between measurement site and motion artifacts in wearable reflected photoplethysmography. J Med Syst 2011; 35: 969-976

9 Spierer DK, Rosen Z, Litman LL, Fujii K. Validation of photoplethysmography as a method to detect heart rate during rest and exercise. J Med Eng Technol 2015; 39: 264-271

${ }^{10}$ Gorny AW, Liew SJ, Tan CS, Müller-Riemenschneider F. Fitbit Charge HR Wireless Heart Rate Monitor: Validation Study Conducted Under Free-Living Conditions. JMIR Mhealth Uhealth 2017; 5: e157

${ }^{11}$ Parak J, Korhonen I. Evaluation of wearable consumer heart rate monitors based on photopletysmography. Conf Proc IEEE Eng Med Biol Soc 2014; 2014: 3670-3673

12 Gillinov S, Etiwy M, Wang R, Blackburn G, Phelan D, Gillinov AM, Houghtaling P, Javadikasgari H, Desai MY. Variable Accuracy of Wearable Heart Rate Monitors during Aerobic Exercise. Med Sci Sports Exerc 2017; 49: 1697-1703

${ }^{13}$ Boudreaux BD, Hebert EP, Hollander DB, Williams BM, Cormier CL, Naquin MR, Gillan WW, Gusew EE, Kraemer RR. Validity of Wearable Activity Monitors during Cycling and Resistance Exercise. Med Sci Sports Exerc 2018; 50: 624-633

${ }^{14}$ Horton JF, Stergiou P, Fung TS, Katz L. Comparison of Polar M600 Optical Heart Rate and ECG Heart Rate during Exercise. Med Sci Sports Exerc 2017; 49: 2600

${ }^{15}$ Shcherbina A, Mattsson CM, Waggott D, Salisbury H, Christle JW, Hastie T, Wheeler MT, Ashley EA. Accuracy in Wrist-Worn, Sensor-Based Measurements of Heart Rate and Energy Expenditure in a Diverse Cohort. J Pers Med 2017; 7 
${ }^{16}$ Delgado-Gonzalo R, Parak J, Tarniceriu A, Renevey P, Bertschi M, Korhonen I. Evaluation of accuracy and reliability of PulseOn optical heart rate monitoring device. Conf Proc IEEE Eng Med Biol Soc 2015; 2015: 430-433

${ }^{17}$ Lin Z, Zhang J, Chen Y, Zhang Q. Heart rate estimation using wrist-acquired photoplethysmography under different types of daily life motion artifact. In: 2015 IEEE International Conference on Communications (ICC). London: IEEE, 2015: 489-494 Im Internet: http://ieeexplore.ieee.org/document/7248369/

${ }^{18}$ Stahl SE, An H-S, Dinkel DM, Noble JM, Lee J-M. How accurate are the wrist-based heart rate monitors during walking and running activities? Are they accurate enough? BMJ Open Sport Exerc Med 2016; 2: e000106

19 Jo E, Lewis K, Directo D, Kim MJ, Dolezal BA. Validation of Biofeedback Wearables for Photoplethysmographic Heart Rate Tracking. J Sports Sci Med 2016; 15: 540-547

${ }^{20}$ Dooley EE, Golaszewski NM, Bartholomew JB. Estimating Accuracy at Exercise Intensities: A Comparative Study of Self-Monitoring Heart Rate and Physical Activity Wearable Devices. JMIR Mhealth Uhealth 2017; 5: e34

${ }^{21}$ Schubert MM, Clark A, Rosa ABDL. The Polar® OH1 Optical Heart Rate Sensor is Valid during Moderate-Vigorous Exercise. Sports Med Int Open 2018; 02: E67-E70

${ }^{22}$ Abt G, Bray J, Benson AC. The validity and inter-device variability of the Apple Watch ${ }^{\mathrm{TM}}$ for measuring maximal heart rate. J Sports Sci 2018; 36: 1447-1452

${ }^{23}$ Claes J, Buys R, Avila A, Finlay D, Kennedy A, Guldenring D, Budts W, Cornelissen V. Validity of heart rate measurements by the Garmin Forerunner 225 at different walking intensities. J Med Eng Technol 2017; 41: 480-485

24 Banister EW. Modeling elite athletic performance. In: Physiological testing of elite athletes. 1991: 403-424

${ }^{25}$ Banister EW, Calvert TW. Planning for future performance: implications for long term training. Can J Appl Sport Sci 1980; 5: 170-176

${ }^{26}$ Manzi V, Iellamo F, Impellizzeri F, D’Ottavio S, Castagna C. Relation between individualized training impulses and performance in distance runners. Med Sci Sports Exerc 2009; 41: 2090-2096

${ }^{27}$ Busso T. Variable dose-response relationship between exercise training and performance. Med Sci Sports Exerc 2003; 35: 1188-1195

${ }^{28}$ Fitzpatrick TB. The validity and practicality of sun-reactive skin types I through VI. Arch Dermatol 1988; 124: 869-871

${ }^{29}$ Wang R, Blackburn G, Desai M, Phelan D, Gillinov L, Houghtaling P, Gillinov M. Accuracy of Wrist-Worn Heart Rate Monitors. JAMA Cardiol 2017; 2: 104-106

${ }^{30}$ Harriss D, Macsween A, Atkinson G. Standards for Ethics in Sport and Exercise Science Research: 2018 Update. Int J Sports Med 2017; 38: 1126-1131 
${ }^{31}$ Bland JM, Altman DG. Statistical methods for assessing agreement between two methods of clinical measurement. Lancet 1986; 1: 307-310

32 Bartko JJ. The Intraclass Correlation Coefficient as a Measure of Reliability. Psychol Rep 1966; 19: 3-11

${ }^{33}$ Koo TK, Li MY. A Guideline of Selecting and Reporting Intraclass Correlation Coefficients for Reliability Research. J Chiropr Med 2016; 15: 155-163

${ }^{34}$ Parak J, Tarniceriu A, Renevey P, Bertschi M, Delgado-Gonzalo R, Korhonen I. Evaluation of the beat-to-beat detection accuracy of PulseOn wearable optical heart rate monitor. In: 2015 37th Annual International Conference of the IEEE Engineering in Medicine and Biology Society (EMBC). 2015: 8099-8102

35 Dondzila C, Lewis C, Lopez J, Parker T. Congruent Accuracy of Wrist-worn Activity Trackers during Controlled and Free-living Conditions. Int J Exerc Sci 2018; 11: 575-584

${ }^{36}$ Le Meur Y, Hausswirth C, Mujika I. Tapering for competition: A review. Sci Sports 2012; 27: $77-87$

37 Terbizan DJ, Dolezal BA, Albano C. Validity of Seven Commercially Available Heart Rate Monitors. Meas Phys Educ Exerc Sci 2002; 6: 243-247

${ }^{38}$ Sarwe A. Heart Rate and Motion Detection on Swimmers Simultaneous monitoring of heart rate and motion of swimmers. 2014; Im Internet: http://studentarbeten.chalmers.se

39 Johnson JM. Exercise in a hot environment: the skin circulation. Scand J Med Sci Sports 2010; 20 Suppl 3: 29-39

${ }^{40}$ Brage S, Westgate K, Franks PW, Stegle O, Wright A, Ekelund U, Wareham NJ. Estimation of Free-Living Energy Expenditure by Heart Rate and Movement Sensing: A Doubly-Labelled Water Study. PLOS ONE 2015; 10: e0137206

41 Andre D, Wolf DL. Recent Advances in Free-Living Physical Activity Monitoring: A Review. J Diabetes Sci Technol 2007; 1: 760-767 


\section{Captions}

\section{$358 \quad$ Figure 1}

359 Examples of HR recordings of Polar H7 (dashed black line) and OH1 (solid grey line) in 360 cyclic (left) and non-cyclic (right). Whereas both signals are mostly identical in running 361 (panel A), Polar OH1 may show a decoupling signal out of the threshold limits (HR dropout, 362 arrow, panel B), in non-cyclic sports (here, kayaking). Panel C illustrates another minor 363 decoupling phenomenon (arrow), potentially impacting values of minimum, maximum and 364 mean HR and therefore TRIMPs.

365

366

Figure 2

367 Bland-Altman plots of $\mathrm{HR}_{\mathrm{OH} 1}$ and $\mathrm{HR}_{\mathrm{H} 7}$ signals for cycling (left) and tennis (right), with bias 368 (thick-dashed black line) and lower and higher values of agreement (thin-dashed black lines). 
371 Session durations, percentage of values out of the 20bpm threshold zone (OH1-OUT), minimum / maximum / mean HR values, bias and LOAs,

Difference $\mathrm{H} 7$ vs. $\mathrm{OH} 1: * \mathrm{P}<0.05, * * \mathrm{P}<0.01, * * * \mathrm{P}<0.001$.

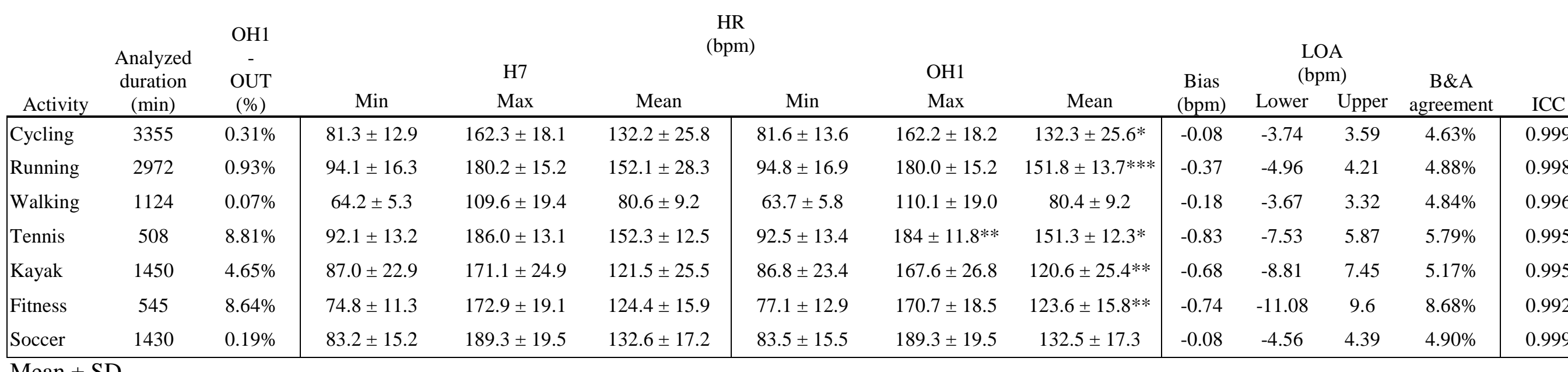


Table 2

383 TRIMP values for each sport, and their mean differences on each session.

384 Difference $\mathrm{H} 7$ vs. OH1: *P $<0.05$, **P $<0.01$, *** $\mathrm{P}<0.001$.

TRIMP

(n.u.)

\begin{tabular}{lccc}
\multicolumn{1}{c}{ Activity } & $\mathrm{H} 7$ & $\mathrm{OH} 1$ & Difference $(\%)$ \\
\hline Cycling & $44.55 \pm 76.32$ & $44.49 \pm 76.25 *$ & $0,67 \pm 0,93$ \\
Running & $74.86 \pm 42.69$ & $74.38 \pm 42.46 * * *$ & $0,71 \pm 0,98$ \\
Walking & $18.53 \pm 12.87$ & $18.38 \pm 12.88$ & -- \\
Tennis & $65.61 \pm 28.25$ & $64.63 \pm 28.72 *$ & $1,90 \pm 2,39$ \\
Kayak & $29.35 \pm 12.79$ & $28.88 \pm 12.94 * *$ & $3,01 \pm 3,17$ \\
Fitness & $42.21 \pm 23.57$ & $41.50 \pm 23.49 *$ & $1,93 \pm 1,87$ \\
Soccer & $71.78 \pm 42.00$ & $71.68 \pm 42.00$ & --
\end{tabular}

385

Mean \pm SD 

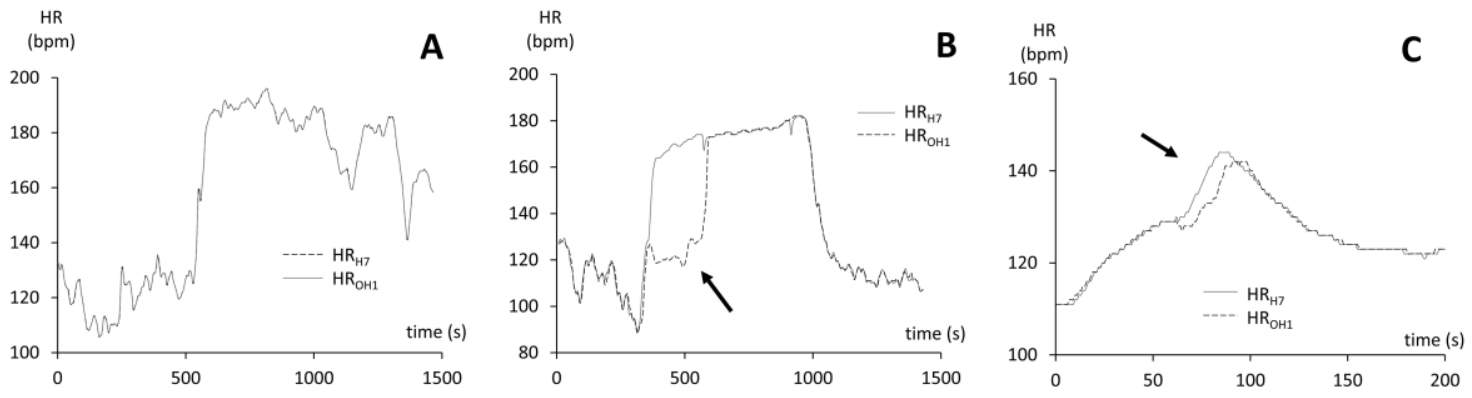

387

Fig. 1
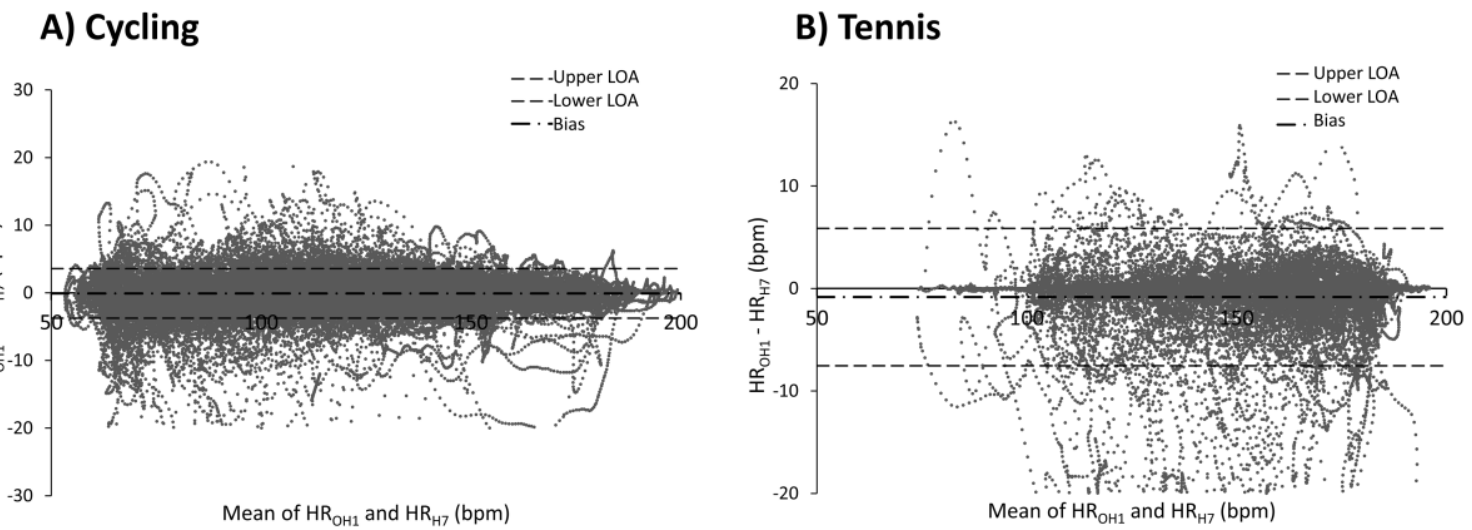

Fig. 2 
391

392 